\title{
Interaction of large Taurid meteoroids with the Earth's atmosphere
}

\author{
N. A. Konovalova ${ }^{\star}$ \\ Institute of Astrophysics Tajik Academy of Sciences, 734042, Bukhoro 22, Dushanbe, Tajikistan \\ and \\ Isaac Newton Institute of Chile, Tajikistan Branch
}

Received 16 October 2002 / Accepted 27 March 2003

\begin{abstract}
Results of the detailed analysis of the luminosity and ablation of bright Taurid, that break up into individual pieces in the Earth's atmosphere due to the aerodynamic pressure are presented. The aim of this analysis is to determine the composition and strength of Taurids on the basis of both photographic observations with small-meteor cameras and cameras with long focal length using the method of instantaneous exposure $\left(\tau_{\exp }=5.6 \times 10^{-4} \mathrm{~s}\right)$. The study was carried out by constructing the theoretical meteor light curve while taking into account its fragmentation into two or more pieces and analyzing the aerodynamic pressure on the meteoroid at the point of splitting. The bulk densities of Taurids were obtained from an analysis of the observed light curves and compared with the strength categories obtained on the basis the data of the aerodynamic pressure at the point of meteoroid splitting. Comparing the resulting values with the known strength properties of various materials lets us conclude that Taurids could be considered structurally fragile bodies that fall into the $a$-strength category, have a density of 2.3 to $2.8 \mathrm{~g} / \mathrm{cm}^{3}$, and can be characterized as carbonaceous chondrites. We discovered a connection between observed low-frequency pulsations of brightness of the splitting Taurids and the flares of individual pieces. We suggested that the observed lateral displacement velocity of the pieces could be caused by the explosive nature of the process of Taurid meteoroid fragmentation.
\end{abstract}

Key words. meteors, meteoroids

\section{Introduction}

In order to study the nature of meteor bodies, researchers analyze the data obtained from meteor observations, which along with applying various models describing interaction of meteoroids with the Earth's atmosphere serve as the basis for determining the material composition of the bodies in question. Despite the fact that over the past few years much work has been done in studying meteor substance and classifying meteoroids (dust components of the Solar system) into different groups based on their composition and structure, this topic remains important. There are several quite successful approaches to this problem that allow establishing of four basic types of material present in meteoroids and fireballs (Wetherill \& ReVelle 1982; Ceplecha \& McCrosky 1976; ReVelle 1983; Sekanina 1983; Ceplecha 1988) namely: Type I - ordinary chondrites; Type II - carbonaceous chondrites; Type IIIA usual cometary material; Type IIIB - very fragile cometary material. Not included is the type of iron-rich material that is observed in iron meteorites. An attempt to identify iron-nickel meteoroids among the fireballs of the Prairie Network (PN) was made by ReVelle \& Ceplecha (1994) who consequently established that seven out of the 287 fireballs were composed of iron. The various body densities of the individual meteors - both the

* e-mail: natalia@ac.tajik.net sporadic and those part of a shower - were determined by analyzing the observed smooth light curves within the framework of the quasi-continuous fragmentation theory. A wide range of meteor density, from $0.4 \mathrm{~g} / \mathrm{cm}^{3}$ up to $7 \mathrm{~g} / \mathrm{cm}^{3}$, was obtained as a result (Babadzhanov 1992a,b, 1993; Novikov et al. 1996). Baldwin \& Sheaffer (1971) have developed the method for analyzing meteor observations data and determining the characteristics of meteor matter, including meteoroids' capability to resist destruction in the atmosphere. They developed a model of fireball fragmentation due to aerodynamic pressure. It is based on the concept of a series of discrete breakup events triggered when increasing aerodynamic pressure exceeds the material's tensile strength. If, however, the aerodynamic pressure exceeds the compressive strength of the material then, according to Baldwin-Sheaffer's model, an extensive fragmentation of the meteoroid will occur. ReVelle (1979) believed that both aerodynamic heating and pressure were responsible for the fragmentation of the fireballs. Ceplecha et al. (1993) developed a dynamic model of the sudden gross-fragmentation for the analysis of photographic observations of the fireballs of the Prairie and European Networks. The result of that analysis was the following classification of fireballs: NF - no-fragmentation; IF - one fragmentation point; MF - many fragmentation points and LA - low accuracy cases. It was also proposed to classify meteoroids into several strength categories: a: $0.8 \mathrm{Mdyn} / \mathrm{cm}^{2}$; 
b: 2.5 , c: $5.3, \mathrm{~d}: 8.0$, e: 11.0 and established their correspondence to the type of material.

Within the framework of the problem of studying the diversity among individual meteoroids inside a meteoroid complex, that is very likely to reflect the diversity among the parent bodies of the complex - the comets this paper analyses and the meteoroids from the Taurid meteor shower. In this analysis, we use the advantages allowed us by the photographic observations done with long-focused meteor cameras MC-75 (D:F = 1:3.5; $F=750 \mathrm{~mm}$ ) equipped with a near-focal rotating shutter that provides for extra-short exposures $\left(5.6 \times 10^{-4} \mathrm{~s}\right)$ when photographing the meteors. This technique allows us to take instant photographs of fireballs brighter than an absolute magnitude of -4 at a high resolution (at the distance of $100 \mathrm{~km}$ from the fireball the spatial resolution is $5 \mathrm{~m}$ in the center of the photoplate and $12 \mathrm{~m}$ at the edge) and presents an opportunity to process the negatives with a high degree of precision.

In this paper, we propose a method of identifying the composition of meteoroids based on analysis of observed features of fireball luminosity in the Earth's atmosphere, which in turn directly depends on the specifics of the meteoroid's destruction. The core of our method consists of modeling a light curve for the meteor that accounts for its splitting into two or more individual fragments that would best correspond to the observed light curve. As a result of comparing the theoretical and the observed light curves we obtain the density $\delta_{0}$ and energy of fragmentation $Q_{\mathrm{f}}$ of the Taurids in question. On the other hand, the observational data on the height and velocity of the meteoroid at the moment of its splitting becomes the basis for calculating the aerodynamic pressure $P_{\text {dyn }}$, from which we infer the strength properties of the Taurids and the material that would fit those properties. The data obtained is compared to the results of the analysis of the observed light curves.

\section{Observations}

\subsection{Double-station photographic observations}

When studying fragmentation of Taurid fireballs we used the observational data obtained from the Meteor Patrol of the Hissar Astronomical Observatory that belongs to Institute of Astrophysics of the Tajik Academy of Sciences. The doublestation photos of the fireballs were taken at Kipchak station located $32 \mathrm{~km}$ from the base point. The double-station photos of meteors obtained with the small-cameras were used mainly to obtain the radiant, height, velocity and mass of the meteors, as well as to calculate their heliocentric orbits. The processing was carried out by a standard computer program (Babadzhanov et al. 1998). The basic data on the atmospheric trajectories of Taurids from 1957 to 1964 was obtained from the Meteor Archive and a published catalogue of orbital elements (Babadzhanov \& Kramer 1963; Babadzhanov et al. 1968).

Sufficiently reliable and precise quantitative estimates of the motion and luminosity of the meteoroids splitting into pieces can be obtained by measuring the images from instantaneous photos taken with meteor cameras equipped with
Uranus-16 long-focused apertures ( $D: F=1: 3.5, F=750 \mathrm{~mm})$. Type 22 film was used in fireball observations, the negative size was $30 \times 30 \mathrm{~cm}$ covering an area of the sky measuring approximately $20 \times 20^{\circ}$, which provided for larger scale imaging and higher resolution. Such an optical system allows us to obtain a reliable estimate of both the linear displacement of the fragments with regards to each other and the lateral displacement at an angle, for they considerably exceed the precision threshold of the optical system used. Today, our photo archives contain a rich collection of instantaneous photographs of 203 fireballs, each having a minimum of 4-5 to a maximum of 92 instantaneous images of individual fireballs. A careful analysis of the instantaneous photographs has shown fireballs splitting into two or more individual fragments for the following showers: Taurids -3 fireballs out of 4 recorded, Perseids -6 out of 25 ; III association and sporadic -6 out of 9 . It is necessary to note that these numbers are at the low end, since not all the abovementioned fireballs are sufficiently bright for their fragments to be distinguishable in the instantaneous photographs. For a comparison, even for the brightest fireballs of the Geminids, $\delta$-Aquarids, and Leonids, instantaneous photographs did not capture any instances of fragmentation.

From the catalogue of instantaneous photographs of meteors, we selected the photographs of three fireball Taurids that show the disintegration of the meteoroids into large fragments: fireballs 643606(1) and 881652 into two fragments, and fireball 852364 into three. At $0.02 \mathrm{~s}$ intervals 32, 39, and 21 instantaneous images of these fireballs have been obtained by the near-focal rotating shutter. Fireballs 643606(1) and 852364 were photographed from two stations and were subsequently processed using the standard computer program. For the ends of each breaks, i.e. for the known instances of time $t$, the values of heights $H$ and velocity $V$ were calculated, and high-quality graphs of velocity as well as reliable deceleration values were obtained. Fireball 881652 was photographed only at one station - the Meteor Patrol of the Hissar Astronomical Observatory and was processed using the method applied to such images. This method accepts the coordinates of the radiant and the velocity as the known values, which is possible if the fireball belongs to a known meteor shower. Of course, in the case of fireball 881652 , we did not have the graphs of velocity and deceleration. The resulting basic data for all the Taurids analyzed in this paper is presented in Table 1, which includes the following: meteor number; $V_{0}$, pre-atmospheric velocity $\left(\mathrm{km} \mathrm{s}^{-1}\right) ; z_{\mathrm{R}}$, zenith distance of the meteor radiant; $H_{\mathrm{F}}$, height of the fragmentation point or strong flare $(\mathrm{km}) ; n_{\mathrm{i}}, n$, number of instantaneous images and measured points along the meteor trajectory respectively; $N_{\mathrm{F}}$, the number of fragments.

\subsection{Photometric data}

When investigating the features of luminosity of Taurid fireballs splitting into fragments, we carried out a detailed analysis of the light curves and the instantaneous images of the three Taurids considered - 643606(1), 852364, and 881652 - taken simultaneously with the small-cameras and the long-focused 
Table 1. Observed and calculated parameters of the Taurid meteors.

\begin{tabular}{|c|c|c|c|c|c|c|c|c|c|c|}
\hline$N / N$ & 643606(1) & 852364 & 881652 & 981119 & $\overline{584412}$ & "643642 & 583815 & 593225 & $643422 a$ & 643302 \\
\hline & \multicolumn{3}{|c|}{ Instant. photo } & \multicolumn{3}{|c|}{ Strong flare } & \multicolumn{4}{|c|}{ Smoothness light curve } \\
\hline$V_{0} \mathrm{~km} \mathrm{~s}^{-1}$ & 33.0 & 33.0 & 29.2 & 29.2 & 29.2 & 29.3 & 30.8 & 29.7 & 32.2 & 30.4 \\
\hline $\mathrm{n}$ inst.im. & 32 & 39 & 21 & - & - & - & - & - & - & - \\
\hline$z_{R}^{\circ}$ & 21.30 & 13.97 & 16.91 & 19.65 & 16.46 & 26.30 & 32.3 & 52.7 & 29.8 & 36.2 \\
\hline$H_{\mathrm{F}} \mathrm{km}$ & 72.4 & 63.6 & 65.5 & 64.3 & 65.8 & 66.0 & - & - & - & - \\
\hline$N_{\text {F piec. }}$ & 2 & 3 & 2 & - & - & - & - & - & - & - \\
\hline n m.p. & 94 & 152 & 44 & 40 & 26 & 122 & 34 & 32 & 74 & 92 \\
\hline$M_{0} \mathrm{~g}$ & 18.2 & 53.8 & 21.2 & 64.2 & 2.1 & 18.2 & 23.0 & 5.25 & 2.54 & 14.6 \\
\hline$M_{\mathrm{F}} \mathrm{g}$ & 6.6 & 21.6 & 8.3 & - & - & - & - & - & - & - \\
\hline$\delta_{0} \mathrm{~g} / \mathrm{cm}^{3}$ & 2.4 & 2.5 & - & - & - & 2.5 & 2.6 & 2.3 & 2.8 & 2.8 \\
\hline$Q_{\mathrm{f}} \mathrm{erg} / \mathrm{g}$ & $4.8 \times 10^{9}$ & $5 \times 10^{9}$ & - & - & - & $5 \times 10^{9}$ & $5.2 \times 10^{9}$ & $4.7 \times 10^{9}$ & $5.8 \times 10^{9}$ & $5.8 \times 10^{9}$ \\
\hline$P_{\mathrm{dyn}} \frac{\mathrm{Mdyn}}{\mathrm{cm}^{2}}$ & 0.510 & 1.492 & 0.952 & 1.764 & .765 & 0.865 & - & - & - & - \\
\hline streng. cat. & $\mathrm{a}$ & $\mathrm{a}$ & $\mathrm{a}$ & $\mathrm{b}$ & $\mathrm{a}$ & $\mathrm{a}$ & - & - & - & - \\
\hline class. & IF & MF & IF & - & - & - & $\mathrm{NF}$ & $\mathrm{NF}$ & $\mathrm{NF}$ & $\mathrm{NF}$ \\
\hline
\end{tabular}

instantaneous cameras. For that purpose, we performed photometric measurements of fireball records with an automated microdensitometer MD-100 using our own method of photometric reduction of instantaneous images (Babadzhanov \& Konovalova 2000). We obtained the light curves of the fireballs in question measuring the different meteor photographs: the rotating shutter photograph, the photograph of the whole fireball, and the instantaneous photograph.

For each measured point along the trajectory we had the following data: the height $H$ in $\mathrm{km}$ and velocity $V$ in $\mathrm{km} \mathrm{s}^{-1}$ obtained by processing the double-station photographs; the brightness $\mathcal{M}$ in absolute magnitude obtained from photometric measurements. Using photometric light curves, we determined the pre-atmospheric mass $M_{0}$ of fireballs in $\mathrm{g}$ as well as the mass $M_{\mathrm{F}}$ of fireballs at the moment of splitting by the following formula:

$M_{0}=2 \int_{t_{\mathrm{b}}}^{t_{\mathrm{e}}} \frac{I}{\tau_{v} V^{3}} \mathrm{~d} t$,

where $t_{\mathrm{b}}$ and $t_{\mathrm{e}}$ denotes the moment of the beginning and of the end of meteor luminosity; $I$ the observed light intensity of the meteor; the luminous efficiency $\tau_{v}$ was expressed as $\tau_{v}=\tau_{0} V$, where $\lg \tau_{0}=-9.28, V$ in $\mathrm{cm} \mathrm{s}^{-1}, \lg I=9.72-0.4 \mathcal{M}$. The resulting values are presented in Table 1 . The observed light curves (the solid line) of those Taurids are shown in Fig. 1, where the $X$-axis gives the height of the fireball above sea level in kilometers and $Y$-axis gives brightness $\mathcal{M}$ in absolute magnitude. Figure 2 shows the photometric profiles of the instantaneous images of the main body and the fragments, where the $X$-axis gives the length $l$ of the image in relative units, and the $Y$-axis gives the value $N$ of the microdensitometer.

Closer to the end of their trajectories, the light curves of fireballs 852364 and 881652 exhibit a multitude of minor, insignificant flares - low-frequency light pulsations. With fireball 852364 , two moderately strong flares are observed before the low-frequency pulsations. Fireball 881652 has a sharp increase of luminosity at the height of $65.4 \mathrm{~km}$, which represents the main flare and lasts approximately $0.06 \mathrm{~s}$, and several

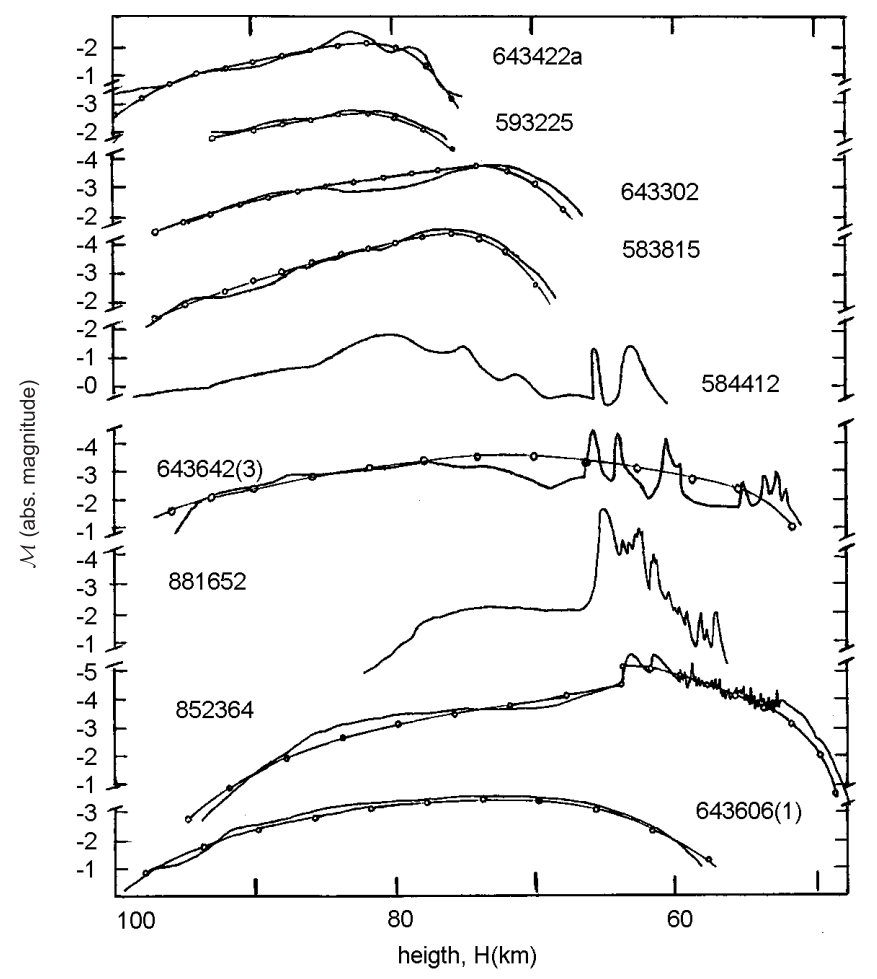

Fig. 1. Observed (solid line) and theoretical (solid line with open symbols) light curves of 9 Taurids. The $y$-axis gives the brightness in stellar absolute magnitudes, normalized to a standard distance of $100 \mathrm{~km}$. The $x$-axis gives the height above the sea level expressed in $\mathrm{km}$.

flares at the final part of the trajectory, their brightness varying from 0.5 to 1.0 absolute magnitude. The sharp increase in fireball luminosity during the main flare could be explained by the sudden increase of the radiating surface, which is possible if at the moment of a meteoroid's splitting into large individual fragments, a lot of smaller fragments with mass of about $10^{-5}-10^{-6} \mathrm{~g}$ are also created. This conclusion is borne out by the fact that the instantaneous photographs of the fireball at the moment of splitting or immediately after contain images of 

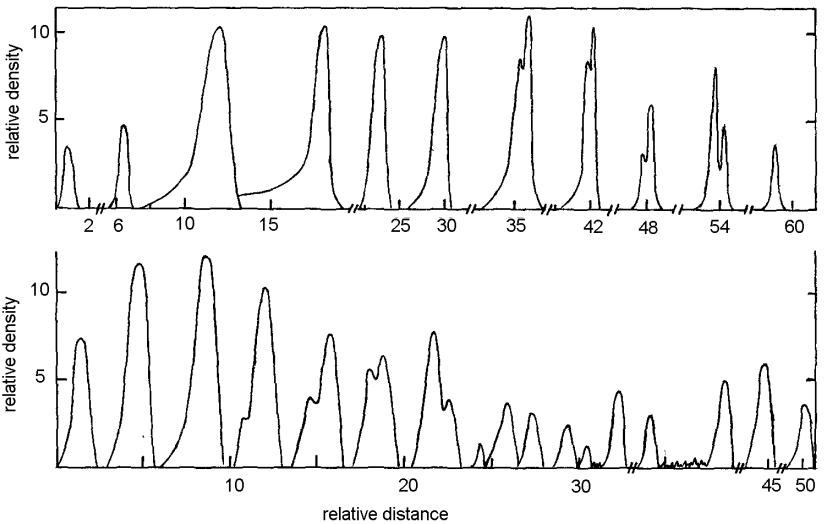

Fig. 2. The part of meteor trajectory of two Taurids: 881653 and 852364 with observed photometric profiles of instantaneous meteor images in which the meteoroid splitting into individual pieces can be seen. The $y$-axis gives the brightness in relative density, the $x$-axis gives the relative distance along the meteor trajectory.

type III, i.e. a disk-shaped coma and a wake of length of more than $100 \mathrm{~m}$. Such meteor wakes as shown in Babadzhanov \& Konovalova (1983) are formed as result of a quasi-continuous fragmentation during which a large number of small particles with a mass of $10^{-5}-10^{-6} \mathrm{~g}$ separate from the main body.

Comparing two photographs of the fragmenting Taurid 881652 obtained with meteor cameras with long focal distance, one of them without a rotating shutter and the other one an instantaneous photo, we determined that the main flare created the image No. 14 with a bright coma and short wake (approx. $280 \mathrm{~m}$ ), which corresponds to type III in the Babadzhanov-Kramer classification system (Babadzhanov \& Kramer 1965). But only $0.02 \mathrm{~s}$ later the next instantaneous image, No. 15, shows a long wake of type IV (over $580 \mathrm{~m}$ ). As shown in Babadzhanov and Konovalova (1983) a long wake of type IV are formed by larger fragments with a mass of $10^{-3}-10^{-2}$ grams that quasi-continuously separate from the main body. This brightest part of the meteor trajectory, the flare, occurs when the main body splits into 2 fragments that are not yet distinguishable in the instantaneous image No. 17; only the wake can be seen. Since the mass of these fragments differs, their deceleration is also different, which makes one fall behind the other, so in the image No. 18 the two fragments are already distinct. The subsequent instantaneous images, Nos. 19, 20, and 21, clearly show the dynamics of the motion and luminosity of these fragments, which not only have a noticeable lag behind one another, but also exhibit a certain degree of lateral displacement.

The comparison and analysis of the fireball images taken with different camera, helped us make another interesting observation: the numerous moderate flares during the final part of the fireball trajectory $(64.0$ to $57.5 \mathrm{~km})$ visible on the norotating-shutter photographs were classified as lowfrequency $\left(N_{\mathrm{p}} \leq 150 \mathrm{~s}^{-1}\right)$ light pulsations on the photos taken with a rotating shutter (Fig. 3). This low-frequency light pulsation, with the help of instantaneous photos, were found to correspond to the flares of the individual fragments. The possibility of a connection between low-frequency pulsation and

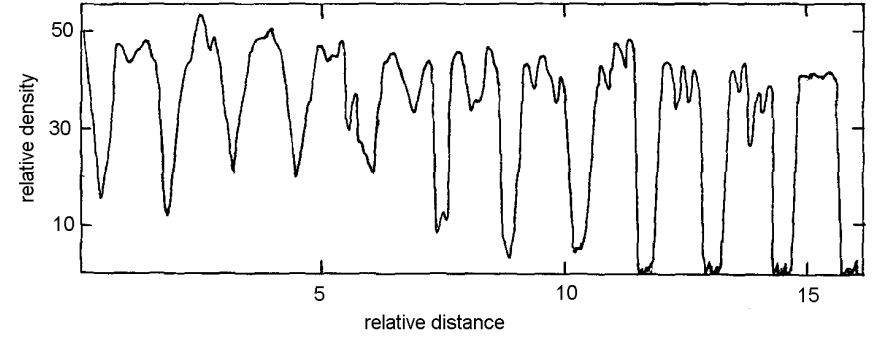

Fig. 3. The part of observed light curve of Taurid 881652. The measured intensity is given as a function of distance. The breaks have been caused by the rotating shutter. The radiation of the pieces and wake can be seen between individual segments.

meteoroid fragmentation is mentioned in Getman (1993). We should note that the cause of low-frequency light pulsation that we discovered through the analysis of instantaneous photographs differs from the nature of the high-frequency pulsations (flickering) observed in bright Geminids (Babadzhanov \& Konovalova 1986, 1987; Halliday 1988). The high-frequency pulsation $\left(N_{\mathrm{p}}=150 \div 400 \mathrm{~s}^{-1}\right)$ with a minor vacillation of brightness $(\Delta \mathcal{M}<0.5 \mathrm{mag})$ observable in bright Geminids originates with the cyclic ejection of the melted surface layer of the meteoroid at a frequency matching the frequency of the observed pulsations.

All three rotating-shutter photos of the splitting Taurids 643606(1), 852364, and 881652 in the final part of their trajectory show an increase in the length of the dashes corresponding to the rotating shutter intervals, to the point where they practically "merge" (Fig. 3). Analysis of the instantaneous photos shows that such "mergers" represent the movement and luminosity of individual fragments acting like independent meteoroids. This observation can be used as direct evidence of a meteoroid's fragmentation into pieces. That allowed us to include in our analysis a few more Taurids that were photographed only with the small-cameras. The same applied to Taurid 981119, photographed from two stations (the High-mountainous Observatory Sanglokh and the Hissar Astronomical Observatory) with all-sky cameras during the observation of Leonid-98. The all-sky cameras were equipped with Zeiss-Distagon ("Fish-Eye") apertures $\left(F / 3.5, F=30 \mathrm{~mm}\right.$, field of vision $180^{\circ}$, diameter of celestical hemisphere on the negative $70 \mathrm{~mm}$ ). Basic information about these meteors is also available in Table 1, and their light curves are presented in Fig. 1.

\subsection{Spectral data}

In this section, we shall consider the spectrum of Taurid 852364, photographed with an instantaneous camera MS-75 that has a spectrum dispersion of $67 \AA \mathrm{mm}^{-1}$. The spectrum consists of 11 individual emission lines and 0-order at the four brightest instantaneous images, taken at the height interval of $60-65 \mathrm{~km}$ with exposure of $3.33 \times 10^{-3} \mathrm{~s}$. The line of the sodium doublet $\mathrm{D}$ NaI, present as the brightest line in the spectrum, is photographed in 26 images down to the end of the meteor. The spectrum of the brightest point of the meteor trajectory at the height of $61.6 \mathrm{~km}$ is presented 


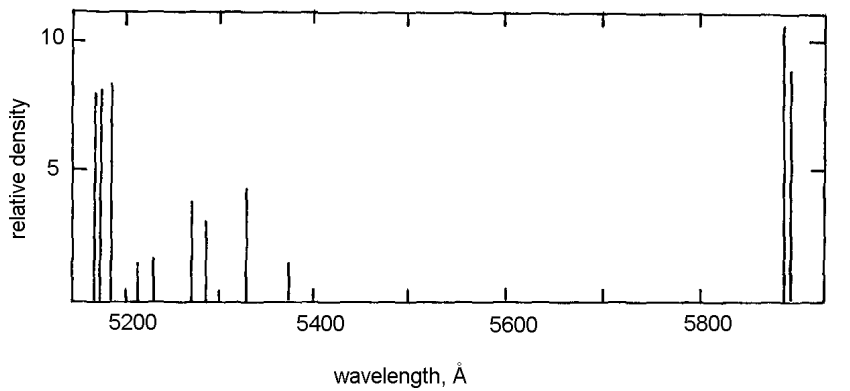

Fig. 4. Lines in the spectrum of Taurid 852364 in the brightest instantaneous meteor image. The units on the $y$-axis are the relative density. The two bright lines on the right are the sodium D line.

in Fig. 4. At this height, the fireball has already broken into 2 fragments and its spectrum is the spectrum of these fragments. As the detailed analysis of the spectrum is outside the scope of this article, we shall limit ourselves to the results of determining the wavelengths of spectral lines and matching them with the corresponding chemical elements. Two tables of atomic spectral lines were used for reference, the multiple table by Moor (1945) and tables of Zajdel' et al. (1977). The wavelength scale was determined by means of known lines in the spectrum.

Thus, in the spectrum of Taurid 852364 the lines of the triplet of magnesium MgI $(5167.32,5172.6,5183.6 \AA)$ and the doublet D NaI $(5889.95,5895.9 \AA)$ were successfully identified. Other lines are the lines of iron FeI: multiple 15

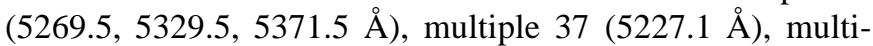
ple $553(5208.6,5229.8,5283.6 \AA)$ and the line CrI multiple 7 (5208.4 $\AA$ ).

\section{Method of calculations}

\subsection{Theoretical light curve of the meteoroid splitting into individual pieces}

In this section we shall concern ourselves with the case of meteoroid ablation in the Earth's atmosphere: the meteoroid with initial mass $M_{0}$ ablating from the initial height $H_{0}$ until the height $H_{\mathrm{F}}$ via quasi-continuous fragmentation during which a large number of small further non-fragmenting particles detach from the parent meteor. After this the splitting of the meteoroid into two pieces with masses $M_{1}$ and $M_{2}$ occurs at the height $H_{\mathrm{F}}$. The mechanism of ablation of these pieces is by quasi-continuous fragmentation until its full disentegration. Such a theoretical model of meteoroid ablation fully conforms to the photographic observations of bright Taurids, where the splitting into individual pieces is directly visible on the instantaneous photographs.

In such a case the following formula for the light intensity $I(t)$ along the meteor trajectory is valid:

$I(t)=I_{0}(t)+I_{1}(t)$,

where $I_{0}(t)$ is the light intensity along the part of the meteor trajectory until the point of meteoroid splitting, $I_{1}(t)$ is the light intensity for the second part of the meteor trajectory after splitting.
The formula for the light intensity, $I_{0}(t)$ at any time $t$ according to the theory of meteoroid quasi-continuous fragmentation (Novikov et al. 1984a,b, 1998) is:

$$
\begin{aligned}
I_{0}(t)= & -\frac{\tau_{v} V_{0}^{2}}{2} \Theta\left(t_{\mathrm{F}}-t\right) \\
& \times \int_{(t-\tau) \Theta\left(t-\tau_{\mathrm{b}}\right)}^{t} N_{\mathrm{f}}\left(t_{0}^{\prime}\right) \frac{\mathrm{d} m_{\mathrm{f}}}{\mathrm{d} t}\left(t ; t_{0}^{\prime}\right) \Theta\left(\tau_{0}-t_{0}^{\prime}\right) \mathrm{d} t_{0}^{\prime},
\end{aligned}
$$

where $\tau_{v}, V_{0}$ are the luminous efficiency and preatmospheric meteoroid velocity; $\Theta(x)$ - Heaviside's function: $\Theta(x)=1$ at $x>0$ and $\Theta(x)=0$ at $x \leq 0$. The function $m_{\mathrm{f}}\left(t ; t_{0}^{\prime}\right)$ is the mass of the fragment, $\tau_{0}$ denotes the lifetime of the parent body to complete fragmentation, $\tau_{\mathrm{b}}$ being the lifetime of the fragment released at the moment of fragmentation beginning, $\tau$ stands for the lifetime of individual fragment counted from $t_{0}^{\prime}$ until its full evaporation, $t_{\mathrm{F}}$ is the instant time of meteoroid splitting.

The number of smaller fragments detached from the parent meteor body per unit time can be expressed as

$N_{\mathrm{f}}\left(t_{0}^{\prime}\right)=-\frac{1}{m_{0}} \frac{\mathrm{d} M}{\mathrm{~d} t_{0}^{\prime}}$

where $M$ denotes the mass of the parent body at the instant time $t_{0}^{\prime}, m_{0}$ are the initial masses of the smaller fragments.

The equations for $\mathrm{d} M / \mathrm{d} t_{0}^{\prime}$ and $\mathrm{d} m_{\mathrm{f}} / \mathrm{d} t$ have been derived by Lebedinets (1980). They read

$\frac{\mathrm{d} M}{\mathrm{~d} t_{0}^{\prime}}=-\frac{\Lambda A M^{2 / 3} \rho V^{3}}{2 Q_{\mathrm{f}} \delta_{0}^{2 / 3}}, \quad \frac{\mathrm{d} m_{\mathrm{f}}}{\mathrm{d} t}=-\frac{\Lambda^{\prime} A^{\prime} m_{0}^{2 / 3} \rho V^{3}}{2\left(Q-Q_{\mathrm{f}}\right) \delta^{2 / 3}}$,

where $\Lambda, A, \delta_{0}$ stand for the heat transfer coefficient, the shapedensity coefficient and the bulk density of the parent body respectively, and $\Lambda^{\prime}, A^{\prime}, \delta$ are analogous quantities valid for fragments, $Q$ denotes the energy of evaporation, while $Q_{\mathrm{f}}$ is the energy of fragmentation, $\rho$ is the atmospheric density.

The light intensity $I_{1}(t)$ is given by following equation for the splitting of meteoroid into two pieces (Babadzhanov et al. 1989):

$I_{1}(t)=-\frac{\tau_{\nu} V_{\mathrm{F}}^{2}}{2} \Theta\left(t-t_{0}^{\prime}\right)\left[\frac{\mathrm{d} M_{1}}{\mathrm{~d} t} \Theta\left(\tau_{01}-t\right)+\frac{\mathrm{d} M_{2}}{\mathrm{~d} t} \Theta\left(\tau_{02}-t\right)\right]$,

where $V_{\mathrm{F}}$ is the velocity of the meteoroid at the moment of splitting, $\tau_{01}$ and $\tau_{02}$ stands for the lifetime of individual pieces counted from the time of splitting until its full ablation, $M_{1}, M_{2}$, are the initial masses of these pieces. The pieces ablate from the time of splitting until full ablation via quasi-continuous fragmentation. The common expressions describing the ablation of pieces along their trajectory is:

$\frac{\mathrm{d} M_{1}}{\mathrm{~d} t_{0}^{\prime}}=\Theta\left(T_{\mathrm{e}}-t\right) \int_{(t-\tau) \Theta\left(t-\tau_{\mathrm{b}}\right)}^{t} N_{\mathrm{f} 1}\left(t_{0}^{\prime}\right) \frac{\mathrm{d} m_{\mathrm{f}}}{\mathrm{d} t}\left(t ; t_{0}^{\prime}\right) \Theta\left(\tau_{01}-t_{0}^{\prime}\right) \mathrm{d} t_{0}^{\prime},(6)$

$\frac{\mathrm{d} M_{2}}{\mathrm{~d} t_{0}^{\prime}}=\Theta\left(T_{\mathrm{e}}-t\right) \int_{(t-\tau) \Theta\left(t-\tau_{\mathrm{b}}\right)}^{t} N_{\mathrm{f} 2}\left(t_{0}^{\prime}\right) \frac{\mathrm{d} m_{\mathrm{f}}}{\mathrm{d} t}\left(t ; t_{0}^{\prime}\right) \Theta\left(\tau_{02}-t_{0}^{\prime}\right) \mathrm{d} t_{0}^{\prime},(7)$ 
where $N_{\mathrm{f} 1}\left(t_{0}^{\prime}\right), N_{\mathrm{f} 2}\left(t_{0}^{\prime}\right)$ denotes the number of small fragments detached from pieces per unit time, $T_{\mathrm{e}}$ stands for the time of termination of the whole meteor event.

The expressions for $\mathrm{d} M_{1} / \mathrm{d} t_{0}^{\prime}, \mathrm{d} M_{2} / \mathrm{d} t_{0}^{\prime}, N_{\mathrm{f} 1}\left(t_{0}^{\prime}\right), N_{\mathrm{f} 2}\left(t_{0}^{\prime}\right)$ and $\mathrm{d} m_{\mathrm{f}} / \mathrm{d} t$ are analogous to Eqs. (3)-(4) valid for individual pieces.

\subsection{Application of theoretical light curves to observation of splitting Taurids}

For the case of ablation of a meteor body in the Earth's atmosphere considered in 3.1, in which splitting into individual fragments takes place, theoretical light curves were modeled using formulas (1-7) to compare with the observed light curves for the Taurids in consideration. For this purpose, we chose Taurids N 643606(1) and N 852364 with high-quality doublestation photographs and instant photographs available. The key parameters for the calculations in Eqs. (1-7) were as follows: the shape-density coefficient for the meteoroid and the main fragments $A=1.5$, for smaller fragments $A=1.21$, specific energy of evaporation $Q=8 \times 10^{10} \mathrm{erg} / \mathrm{g}$. The dependence of the luminous efficiency $\tau_{v}$ upon the velocity of the meteor $V$ in calculating the theoretical light intensity $I(t)$, as well as the mass of the meteoroid $M_{0}$ upon the observed light intensity of the meteor was expressed as $\tau_{v}=\tau_{0} V$, where $\lg \tau_{0}=-9.28$, $V$ in $\mathrm{cm} \mathrm{s}^{-1}, \lg I=9.72-0.4 \mathcal{M}$. This approach allowed us to avoid the uncertainty related to the choice of value for the luminous efficiency when comparing the observed and theoretical light curves.

For the smaller fragments, the heat transfer coefficient $\Lambda^{\prime}$ was assumed to be equal to 1 ; for the meteoroid and the main pieces with size less than $1 \mathrm{~cm}$ it was equal to 0.5 ; and for those larger than $1 \mathrm{~cm}$ (in view of the strong obstruction effect, as a result of which the heat transfer coefficient over the trajectory can change almost by a degree) it is equal to 0.40.04 (Bronshten 1981). The initial smaller fragment mass $m_{0}$ was assumed identical and equal to $10^{-5} \mathrm{~g}$, while the masses of the meteoroid and of the pieces were obtained through photometric measurements of meteor images. As the composition of the Taurid in question is not known a priori, the density of the meteoroid $\delta_{0}$ and of its fragments $\delta$ and the specific energy of fragmentation $Q_{\mathrm{f}}$ were obtained by trial and error by searching for the theoretical light curve that would best match the observed one. The data of densities $\delta_{0}$ and of energy of fragmentation $Q_{\mathrm{f}}$ for different types of meteoroids (Lebedinets 1980, Table 1) were used in the calculations, which were carried out by computer at a step equal to $0.1 \mathrm{~g}$.

The results are presented in Fig. 1 where the solid line represents the observed light curves of the splitting Taurids $\mathrm{N} \mathrm{643606(1)} \mathrm{and} \mathrm{N} 852364$ while the solid line with open symbols shows the theoretical light curves, calculated with Eqs. (1-7). The data on meteoroid density $\delta_{0}$ in $\mathrm{g} / \mathrm{cm}^{-3}$ and specific energy of fragmentation $Q_{\mathrm{f}}$ in $\mathrm{erg} / \mathrm{g}$, obtained by comparing the theoretical light curve calculated using our model with the observed light curve, are also present in Table 1.

\subsection{The regular light curves of the Taurids}

To compare the data on the determined type of composition for the splitting Taurids in question, we have analyzed the regular light curves of several Taurids that we had selected from our collection of high-quality double-station meteor records with reliable processing. At the present, it is a firmly established belief that the fragmentation of meteor bodies in the Earth's atmosphere is the most common form of ablation. This is why we chose to analyze the observed regular light curves of the selected Taurids within the framework of a quasi-continuous fragmentation model.

The method of calculation of the theoretical light curves was the same as in the case described in Sect. 3.2, the only difference being that the theoretical intensity of light $I(t)$ was calculated along all meteor trajectories, since they did not split into individual fragments. Figure 1 shows the observed light curves of Taurids NN 583815, 593225, 643433a and 643302a as well as the theoretical light curves calculated for them. The data on the obtained meteoroid density $\delta_{0}$ and energy of fragmentation $Q_{\mathrm{f}}$ are also presented in Table 1.

\subsection{Splitting due to aerodynamic pressure}

The splitting of meteor bodies in the Earth's atmosphere occurs due to the fracturing pressure caused by the aerodynamic pressure applied by the oncoming stream of air. Analyzing the impact of aerodynamic pressure on the movement and ablation of the meteoroid can be used to determine the physical properties and the substance composing the bodies investigated.

The fracturing pressure affecting the meteoroid during its movement through the Earth's atmosphere depends on its velocity $V$. Therefore, the more precisely the meteoroid's velocity is determined at the height of splitting as well as the value of the height itself, the more reliable will be the data on the aerodynamic pressure acquired by processing the meteor records. Particularly successful in this regard are the photos of meteors taken with long-focused cameras by the method of instantaneous exposures. Through astrometric and photometric measuring of the instantaneous photos, we obtained the data on the height $H_{\mathrm{F}}$ of the meteoroid's splitting into fragments, velocity $V_{\mathrm{F}}$ at the moment of splitting, number of pieces $N_{\mathrm{F}}$ and their mass $M_{\mathrm{F}}$, and also the information on whether the fragmentation was singular, i.e. only at one point of the trajectory, or multiple. Thus, on the basis of the data from double-station and instantaneous meteor photos, we calculated the dynamic pressure at the height of the meteoroid's splitting

$P_{\mathrm{dyn}}=\Gamma \rho_{\mathrm{a}} V^{2}$

where the drag coefficient $\Gamma=1$, and the air density $\rho_{\mathrm{a}}$ is taken from the tables of the standard seasonal atmosphere (CIRA 1972). The results are shown in Table 1 . We analyzed them to determine whether the Taurids under study belonged to any of the strength categories proposed in Ceplecha at al. (1993). The results of that analysis are presented in Table 1.

Figure 5 shows the velocity diagrams for the selected Taurids. The lines representing the constant aerodynamic pressure were calculated assuming the drag coefficient $\Gamma=1$. 


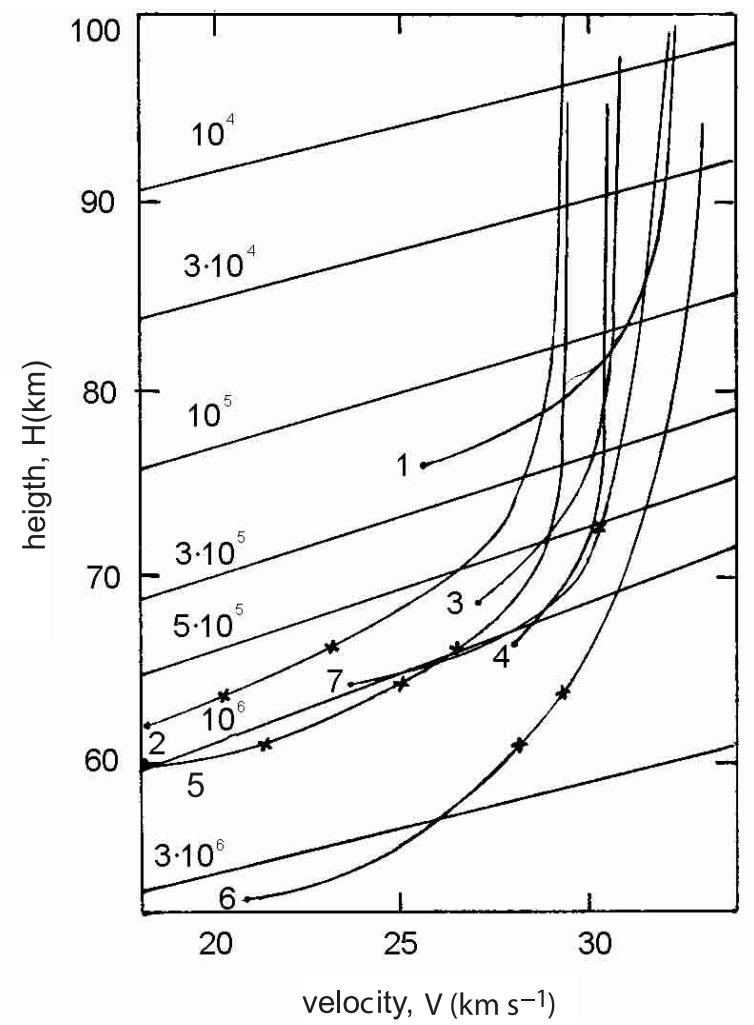

Fig. 5. The height-velocity diagram for the several Taurids. The "+”" symbols marks the point of the fireball splitting or the strong flare. The lines running from the top right to the bottom left are lines of constant aerodynamic pressure calculated from Eq. (8) with a drag coefficient $\Gamma=1.1-643422 \mathrm{a}, 2-584412,3-583815,4-643302$, 5 - 643642(3), 6-852364, 7 - 643606(1).

This drag coefficient corresponds to the one adopted in Sects. 3.2 and 3.3. Figure 5 shows the tendency of Taurid fireballs to split into pieces under aerodynamic pressures of about 0.5 to $1.1 \mathrm{Mdyn} / \mathrm{cm}^{2}$. This result correlates well with the bulk densities of the researched Taurids determined from the analysis of light curves in Sects. 3.2 and 3.3. Based on this, we concluded that Taurids are mostly carbonaceous chondrites with bulk densities of about $2.3-2.8 \mathrm{~g} / \mathrm{cm}^{3}$. The data on the bulk density, specific energy of fragmentation and dynamic pressure, obtained by analyzing the light curves and the heights at which the Taurids in question fractured, presents the details on strength properties of individual Taurid meteoroid and the structure and composition of their matter.

\subsection{Lateral displacement of pieces of split fireballs}

The instantaneous photos of Taurids 852364 and 881652 distinctly show lateral displacement of the pieces formed as a result of sudden splitting of these fireballs. The velocity $U_{\perp \text { obs }}$ of the lateral displacement of the pieces, obtained by measuring these records, appears to be equal to $132 \mathrm{~m} \mathrm{~s}^{-1}$ and $77 \mathrm{~m} \mathrm{~s}^{-1}$ accordingly. We note that these values of $U_{\perp \text { obs }}$ are the lower bound of the average velocity of lateral displacement since we did not take into account the angle of observation.

As noted in Nemchinov et al. (1999), the simple destruction of the meteoroid - i.e. the loss of durability as a result of the aerodynamic pressure - in itself cannot release enough energy to account for the observed velocity of fragment displacement. We can estimate the velocity $U_{\perp}$ of the lateral displacement of pieces under simple aerodynamic pressure by using the equation proposed by Nemchinov et al. (1999):

$U_{\perp}=V \sqrt{\rho_{\mathrm{a}} / \delta_{0}}$

where $V$ and $\delta_{0}$ are the velocity and the density of the meteor body respectively, and $\rho_{\mathrm{a}}$ is the air density at the height of fracturing. Considering that the average height of splitting for the Taurids in question is $H_{\mathrm{F}}=65 \mathrm{~km}$, where air density $\rho_{\mathrm{a}}$ is equal to $1.718 \times 10^{-7} \mathrm{~g} / \mathrm{cm}^{3}$, meteoroid velocity $V=27 \mathrm{~km} \mathrm{~s}^{-1}$, and Taurid average density $\delta_{0}=2.5 \mathrm{~g} / \mathrm{cm}^{3}$, as given in Table 1 , we conclude that the lateral velocity $U_{\perp}$ is about $7 \mathrm{~m} \mathrm{~s}^{-1}$. Therefore, the observed lateral displacement velocity $U_{\perp \text { obs }}$ of the pieces exceeds the velocity obtained from Eq. (9) by more than an order of magnitude. From that, we conclude that the observed lateral displacement velocity of the pieces could be caused by an explosive process that resulted in fracturing of the meteoroid.

The spectrum obtained from fragments of fractured fireball 852364 contains bright lines of the MgI triplet (see Fig. 4). Magnesium, by virtue of its explosive properties, can be one of the leading causes of the meteoroid fracturing. It can be considered the "trigger" initiating the process of explosion when the increasing aerodynamic pressure exceeds the critical strength of the meteor body. In addition to this, the spectrum of the fracturing fireball 770533 obtained from the instantaneous images taken with long-focused cameras also contained strong lines of magnesium (Babadzhanov et al. 1979), as did the fracturing fireball EN 151068 (Borovicka 1993).

\section{Conclusion}

The main results are the following.

Research on the luminosity and ablation of Taurid fireballs was conducted by double-station photographic observation using "small" and long-focused cameras and the instantaneous exposure technique. The study was carried out by the combined method of constructing the theoretical meteor light curve while taking into account its fragmentation into two or more pieces and analyzing the aerodynamic pressure on the meteoroid at the point of splitting.

For the Taurids with precise dynamic data at the height of the disintegration available, we calculated the aerodynamic pressure $P_{\text {dyn }}=\Gamma \rho_{\mathrm{a}} V^{2}$, which fell into the range of 0.51 to $1.76 \mathrm{Mdyn} / \mathrm{cm}^{2}$. Comparing the resulting values with the known strength properties of various materials lets us conclude that Taurids could be considered structurally fragile bodies that split along fissure lines into large pieces due to the aerodynamic pressure of the incoming stream of air. The Taurids that fractured into individual fragments fall into the $a$ strength category (Ceplecha et al. 1993), have density of 2.3 to $2.8 \mathrm{~g} / \mathrm{cm}^{3}$, and can be characterized as carbonaceous chondrites. Thus, they belong to the Type II meteoroids according to the classification in Ceplecha et al. (1993) for which the presence of one or several points of fragmentation is typical. 
The lateral displacement velocity $U_{\perp}$ of the pieces at the height of splitting under the simple aerodynamic pressure for the Taurids was calculated. The observed lateral displacement velocity $U_{\perp \text { obs }}$ obtained from measuring the instantaneous images of these fireballs appeared higher by more than an order of magnitude than the calculated velocity. From this we concluded that the observed lateral displacement velocity of the pieces could be caused by the explosive nature of the process of meteoroid fragmentation.

The statistical data received from observations indicate that the Taurids most likely to fracture are the large ones, with a mass of a few tens of grams, that enter the Earth's atmosphere at a small zenith angle $\left(z_{\mathrm{R}}<20^{\circ}\right)$. The spectrum of split of the Taurids contains strong lines of magnesium. The presence of magnesium in the composition of Taurids can be considered as the leading cause, due to its explosive properties, of the sudden fracturing of the meteoroid into large individual fragments. The observed light curves of such Taurids have one or several strong $(\mathcal{M}=-3.5$ to $-5.5 \mathrm{abs} \mathrm{mag})$ flares.

Using photographic observations, we discovered and analyzed the specific features of luminosity of bright Taurids at the final part of a meteor trajectory, that are expressed in multiple low-frequency $\left(N_{\mathrm{p}}<150 \mathrm{~s}^{-1}\right)$ pulsation of brightness. On the basis of the data acquired by analyzing the instantaneous photos of these fireballs, we concluded that the low-frequency brightness pulsations are flares radiated by individual pieces formed as a result of aerodynamic disintegration of the main meteoroid. The observed elongation and the following "merge" of the rotating shutter dashes is caused by the luminosity of the large fragments moving one after another with a certain lag due to the difference in the mass and therefore the difference in deceleration. On the basis of these data, we came to the conclusion that a sharp amplification of brightness (a flare) followed by low-frequency pulsations observed in meteor light curves, along with the "merging" of rotating shutter breaks in the photographs of these meteors, can be considered an indirect indication of meteoroid splitting.

Acknowledgements. I thank prof. P.B. Babadzhanov and the referee Dr. D. Asher for help in improving the article and also the staff of the Meteor department for their help during the double-station observations. I gratefully acknowledge prof. Z. Ceplecha and Dr. J. Borovicka for giving me the photoplates for our observations of fireballs and also M.V. Yereshenko for the translation this paper from Russian into English.

\section{References}

Babadzhanov, P. B., \& Kramer, E. N. 1963, Methods and some results of photographic researches of meteors, Moscow, AN SSSR

Babadzhanov, P. B., \& Kramer, E. N. 1965, Astron. J., 42, 660

Babadzhanov, P. B., Getman, T. I., Zausaev, A. F., et al. 1968, Bulletin of the Institute of Astrophysics, Tajik Acad. Sci., 49, 3

Babadzhanov, P. B., Getman, V. S., Zolova, O. F., et al. 1979, Astron. Vestnik, 13, 243

Babadzhanov, P. B., \& Konovalova, N. A. 1983, Doklady Academii Nauk Tajik SSR, 26, 494
Babadzhanov, P. B., \& Konovalova, N. A. 1986, Doklady Academii Nauk Tajik SSR, 29, 262

Babadzhanov, P. B., \& Konovalova, N. A. 1987, Publ. Astron. Inst. Czechosl. Acad. Sci., 67, 189

Babadzhanov, P. B., Novikov, G. G., \& Konovalova, N. A. 1989, Astron. Vestnik, 23, 277

Babadzhanov, P. B., \& Makhmudov, N. 1992, Doklady Academii Nauk Tajik SSR, 35, 24

Babadzhanov, P. B., \& Makhmudov, N. 1992, Comet. circular, 426

Babadzhanov, P. B. 1993, in Meteoroids and their parent Bodies, ed. J. Stohl, \& I. P. Williams, Astron. Inst. of the Slovak Acad. Sci., Bratislava, 295

Babadzhanov, P. B., Zubareva, T. I., Konovalova, N. A., et al. 1998, Bulletin of the Institute of Astrophysics, Tajik Acad. Sci., 82, 16

Babadzhanov, P. B., \& Konovalova, N. A. 2000, Bulletin of the Institute of Astrophysics, Tajik Acad. Sci., 84, in press

Babadzhanov, P. B. 2002, A\&A, 384, 317

Baldwin, B., \& Sheaffer, Yv. 1971, J. Geoph. Res., 76, 4653

Halliday, I. 1988, Icarus, 76, 2

Borovicka, J. 1993, A\&A, 279, 627

Bronshten, V. A. 1981, Fizika meteornykh yavlenij, Nauka, Moscow; English translation: Physics of Meteoric Phenomena (Dordrecht: Reidel, 1983)

Ceplecha, Z., \& McCrosky, R. E. 1976, J. Geophys. Res., 81, 6257

Ceplecha, Z. 1988, Bull. Astron. Inst. Czechosl., 39, 221

Ceplecha, Z., Spurny, P., Borovicka, J., et al. 1993, A\&A, 279, 615

Cospar International Reference Atmosphere 1972 (Berlin: Acad.Verlag, 1972)

Getman, V. S. 1993, in meteoroids and their parent bodies, ed. J. Stohl, \& I. P. Williams, Astron. Inst. of the Slovak Acad. Sci., Bratislava, 307

Lebedinets, V. N. 1980, Pyl' v Verkhnej Atmosfere i Kosmicheskom Prostranstve, Meteory, Gidrometizdat, Leningrad, 248

Moore, Ch. E. 1945, A Multiplet Table of Astrophysical Interest, Contrib. Princeton Univ. Obs. 20

Nemchinov, I. V., Kuzmicheva, M. Yu., Shuvalov, V. V., et al. 1999, in Evolution and Source Regions of Asteroids and Comets, ed. J. Svoren, E. M. Pittich, \& H. Rickman, Astron. Inst. Slovak Acad. Sci., Tatranska Lomnica, Proc. IAU Coll., 173, 51

Novikov, G. G., Lebedinets, V. N., \& Blokhin, A. V. 1984, Pis'ma v A $\mathrm{Zh}, 10,71$

Novikov, G. G., Lebedinets, V. N., \& Blokhin, A. V. 1984, Pis'ma v A Zh, 10, 785

Novikov, G. G., \& Konovalova, N. A. 1995, Astron. Vestnik, 29, 547

Novikov, G. G., Pecina, P., \& Konovalova, N. A. 1996, A\&A, 306, 991

Novikov, G. G., Pecina, P., \& Konovalova, N. A. 1998, A\&A, 329, 769

ReVelle, D. O. 1979, J. Atmos. Terr. Phys., 41, 453

ReVelle, D. O. 1983, Meteoritics, 18, 386

ReVelle, D. O., \& Ceplecha, Z. 1994, A\&A, 292, 330

Sekanina, Z. 1983, Astron. J., 88, 1382

Spurny, P., \& Borovicka, J. 1999, in Evolution and source Regions of Asteroids and Comets, ed. J. Svoren, E. M. Pittich, \& H. Rickman, Astron. Inst. Slovak Acad. Sci., Tatranska Lomnica, Proc. IAU Coll., 173, 163

Tsvetkov, V. I., \& Skripnik, A. Ya. 1991, Astron. Vestnik, 25, 364

Wetherill, G. M., \& ReVelle, D. O. 1982, in Comets, ed. L. L. Wilkening (Tucson: University of Arizona), 297

Zajdel', A. N., Prokof'ev, V. K., Rajskij, S. M., et al. 1977, Tablitsy spectral'nykh linij (Tables of spectral lines), (Moscow: Nauka) 